\title{
CONOCIMIENTO PROFESIONAL Y REFLEXIÓN SOBRE LA PRÁCTICA DEL PROFESOR UNIVERSITARIO
}

\author{
Irving Daniel Martínez-Ayala* \\ Margarita Armenta Beltrán \\ Héctor Manuel Jacobo García
}

\section{RESUMEN}

Esta investigación presenta los resultados parciales de la implementación de un programa de desarrollo profesional realizado con profesores de la Facultad de Ciencias de la Tierra y el Espacio (FACITE) de la Universidad Autónoma de Sinaloa (UAS). Su propósito es favorecer la reintroducción del profesor como sujeto de saber y evidenciar con ejemplos, como el presente estudio, que contribuyan a fortalecer la epistemología de la práctica y la teoría general de las profesiones, el caso de los educadores. Los resultados indican que la inclusión de programas de acompańamiento entre pares genera un mayor nivel de complejidad en la reflexión profesional realizada por el docente universitario y pone de relieve distintas dimensiones o saberes de su conocimiento profesional, aunque las dificultades mayores se presentan en el ajuste a la reflexión técnica para superar las teorías intuitivas que fundamentan su quehacer docente.

PALABRAS ClAVE: epistemología de la práctica, teoría general de las profesiones, conocimiento profesional, reflexión profesional, formación profesional situada, profesor reflexivo.

\section{PROFESSIONAL KNOWLEDGE AND REFLECTIVE PRACTICE \\ OF THE UNIVERSITY PROFESSOR}

\section{Abstract}

This research presents the partial results of the implementation of a professional development program carried out with professors of the Faculty of Earth and Space Sciences (FACITE) of the Autonomous University of Sinaloa (UAS). Its purpose is to favor the reintroduction of the teacher as a subject of knowledge and to demonstrate with examples, such as the present study, that contribute to strengthen the epistemology of the practice and the general theory of the professions, the case of educators. The results indicate that the inclusion of accompaniment programs among peers, generates a greater level of complexity in professional reflection made by the university teacher and highlights different dimensions or knowledge of their professional knowledge, although the greatest difficulties arise in the adjustment to the technical reflection to overcome the intuitive theories that base their teaching task. KEYWORD: epistemology of practice, general theory of the professions, professional knowledge, professional reflection, professional training situated, reflexive teacher. 


\section{INTRODUCCIÓN}

El desempeño docente del profesor universitario en la educación superior ha generado múltiples investigaciones cuyo objeto de estudio ha sido la enseñanza universitaria, principalmente las que se refieren a la indagación sobre qué y cómo piensan, cómo construyen su conocimiento y cómo aprenden a enseñar los profesores (Gamboa, 2017).

Se ha dicho que la práctica educativa del profesorado universitario -como los de otros niveles educativos- se da en un contexto de aprendizaje en el que su quehacer docente y la socialización de experiencias con otros colegas, entre otros factores, dé lugar a una formación pedagógica informal (Hurtado, Sema y Madueño, 2015). También se afirma que, en su mayoría, el profesor universitario accede a la docencia sin formación pedagógica y didáctica previa, emigrando desde otros espacios profesionales o de investigación (Gamboa, 2017).

Es por eso por lo que se hace necesario profundizar el conocimiento del profesor universitario, para lo cual se plantean algunas interrogantes, tales como ¿cuál es el conocimiento profesional del profesor universitario?, ¿cómo influye el acompańamiento entre pares para mejorar la práctica docente?, ¿cuáles son los saberes de los profesores universitarios al reflexionar en comunidad de práctica docente?

Este estudio se posiciona dentro del campo de investigaciones que ha dado lugar a lo que en un principio se denominó como el paradigma del pensamiento del profesorado (Imbernón, 2004), campo de estudios con un enfoque cognitivo que de acuerdo con Shavelson y Stern (1981) parte de dos premisas: la primera, aceptar al profesor como un sujeto reflexivo y racional, que puede tomar decisiones y emitir juicios de valor al igual que otros profesionistas; la segunda, que son los pensamientos del profesor los que guían su conducta al momento de proceder en su intervención docente.

El punto de partida de este programa de investigación era pues entender que para generar comprensión sobre el comportamiento de los profesores es necesario indagar sobre sus procesos mentales, sus contenidos, métodos y procedimientos (Pérez y Gimeno, 1988). Aunado a lo anterior, es pertinente señalar que desde ese entonces ya se tenía en cuenta que el profesor también posee un conjunto de creencias, rutinas y teorías implícitas que construye en su práctica docente, esto forma parte de esa laguna existente entre las explicaciones sobre la relación del pensamiento y la acción (Angulo 1999; Marrero, 2009).

El enfoque meramente cognitivo de este campo de investigación evidenció al paso del tiempo ciertas dificultades de orden teórico y metodológico por el énfasis

*E-mail: irvingmartinez.face@uas.edu.mx. Licenciado en Ciencias de la Educación por la Universidad Autónoma de Sinaloa. Estudiante de posgrado en el Programa Maestría en Educación en la Facultad de Ciencias de la Educación de la Universidad Autónoma de Sinaloa, México. Investiga las teorías implícitas, los procesos de reflexión y el conocimiento profesional docente de los profesores universitarios. 
cognitivo que descuidó variables de carácter emocional y contextual, estas dificultades generaron un nuevo interés por indagar no sólo cómo piensa el docente, sino también qué piensa y por qué piensa de esa manera (Gutiérrez, 2012). Siguiendo a Marcelo (2005) la investigación sobre el pensamiento del profesor dio una transición al preocuparse más por el conocimiento del profesor, fue un nuevo interés que se vio motivado por el movimiento reflexivo en educación que tuvo sus claros inicios en los aportes de Dewey, pero que con los trabajos de Schön $(1992,1998)$ principalmente se consolidó una creciente influencia contextual que considera a los profesores como profesionales que desarrollan una epistemología o conocimiento de la práctica, conocimiento que merece ser investigado.

Diversos estudios surgieron en esta línea renovada sobre el conocimiento profesional de los profesores, estos estudios han expuesto diferentes propuestas teóricas en las cuales diversifican el conocimiento del profesor en distintos saberes o dimensiones (Elbaz, 1981; Marcelo, 2005; Pérez, Soto y Serván, 2015; Porlán, Rivero y Martín, 1997; Shulman, 1987; Tardif, 2004), encontrando que el conocimiento profesional del profesor puede tener un carácter práctico, personal, implícito, entre otros; sin embargo, es pertinente señalar lo que Rodríguez y Alamilla (2018) exponen:

Independientemente del término empleado, lo destacable aquí es el reconocimiento de la docencia como una actividad en la que el pensamiento y la acción se vinculan para dar lugar al conocimiento profesional. De modo que este se concibe como conformado, no solo a través de saberes de tipo teórico, sino también por medio de saberes experienciales o prácticos (p. 3).

Atendiendo las características del contexto universitario, el conocimiento del profesor del nivel superior es un conocimiento práctico, pues orienta a guiar la acción educativa y además surge de la experiencia práctica (Dávila, Leal, Comelin, Parra y Varela, 2013); sin embargo, este tipo de conocimiento es tácito y en ocasiones no verbalizable (Clarà y Mauri, 2010), pues de acuerdo a su proceso de construcción informal se ve cargado de intuición, intenciones y emociones (Pérez-Gómez, 2010). En este sentido se reconoce que tiene una dimensión de carácter implícito (Marrero, 1993), pues el profesor con base en su síntesis de experiencias de prácticas pedagógicas construidas, decisiones, acciones, rutinas y hábitos realizados en un escenario cultural escolar ha constituido una serie de teorías implícitas que guían la práctica educativa y dan lugar a su conocimiento práctico (Marrero, 2009).

En este trabajo se percibe el conocimiento profesional en el sentido complejo de Morín (2002); se constituye en una emergencia cuya construcción, por mencionar algunas relaciones, es el resultado del entramado entre su historia formativa en su trayecto inicial, las interacciones de los procesos de enseńanza y aprendizaje, así como la reflexión de los profesores. Asimismo, hay una identificación con los planteamientos teóricos del movimiento reflexivo en la educación de Dewey (1989) y se adhiere a la perspectiva que hace referencia a la generación de conocimiento profesional a partir de la retroacción que realiza el profesor durante y después de la acción (Schön, 1998). 
En lo que concierne al movimiento reflexivo en la educación, John Dewey es un clásico, pionero y referente; su obra Cómo pensamos (1989) ha sido punto de partida de diversos autores para hablar y justificar sobre el pensamiento y la formación reflexiva en educación. Pertinente es partir de su conceptualización, lo que constituye el pensamiento reflexivo es el examen activo, persistente y cuidadoso de toda creencia o supuesta forma de conocimiento a la luz de los fundamentos que la sostienen y las conclusiones a las que tiende (Dewey, 1989, p. 24).

La reflexión de los profesionales de la educación tiene una finalidad, es un proceso consciente que implica un análisis retroactivo y meticuloso sobre las acciones que se realizaron, se están realizando y se realizarán. Esta retroalimentación constante lleva a los profesores a replantear sus prácticas pedagógicas y a hacer explícito su conocimiento puesto en práctica. En esta misma perspectiva se inscribe el planteamiento de Schön (1998) sobre cómo piensan los profesionales, y marca una distinción cuando propone una diversificación del proceso reflexivo en dos fases: reflexión sobre la acción y reflexión en la acción.

En esta línea, el profesional que reflexiona sobre su quehacer docente, (Jacobo, 2009), adherido a la compresión sistémica y compleja (Johansen, 1992; Morín, 2002) reconoce que la reflexión profesional es un ejercicio autorregulatorio orientado a mejorar los procesos del ejercicio docente, en el cual la metacognición juega un papel importante.

Para efectos de esta investigación se retoma la propuesta realizada por Jacobo (2009) en El Profesionalismo Integrado, sobre la reflexión profesional en comunidades de práctica docente, en las dimensiones práctica, técnica y social. Una comunidad de práctica está constituida por un grupo de educadores que deciden dar lo mejor de sí para cooperar y colaborar en la formación y desarrollo profesional de sus colegas; sus objetos son diversos. Uno de ellos es tomar conciencia del repertorio de rutinas de trabajo pedagógico con estrategias didácticas efectivas; otro, la reflexión crítica sobre el trabajo profesional para hacer explícito el conocimiento tácito del que derivan; y adquirir y desarrollar toda clase de competencias que contribuyan a plantear, explicar, comprender y resolver problemas profesionales de manera individual y colectiva (Jacobo et al., 2005).

En este caso, el propósito es hacer explícito el conocimiento tácito a través de un ejercicio de reflexión profesional para evidenciar los saberes que componen el conocimiento profesional mediante el acompañamiento entre pares.

Cabe destacar que, de acuerdo con Jacobo et al. (2005), el acompañamiento se inserta en una red de relaciones colaborativas caracterizada por su horizontalidad, retroacción y recursividad y está determinado por la humanización de las relaciones sociales. Además, supone una interacción generosa y de calidad entre personas con un desarrollo desigual pero unidas con una misma intención: la adquisición y desarrollo de competencias profesionales. Vale decir que la reflexión profesional adquiere significado en el acompañamiento en la profesión, el cual demanda del profesional descentrarse de sí, desarrollar empatía y cierto perspectivismo emocional y cognitivo que favorezcan la comprensión del acompañado.

De acuerdo con Jacobo (2009), la reflexión profesional se compone de tres dimensiones: práctica, técnica y social; sin embargo, el hecho de que ésta sea de una 
o de otra manera no depende sólo de que el educador sepa combinarla, sino de que también sea sensible a lo que demandan los contextos. A continuación, se exponen sus características principales:

a) Reflexión práctica. Se centra en la práctica docente, su finalidad es resolver los incidentes críticos que se presentan durante el proceso de enseñanza, implica que los profesores analicen y cuestionen su trabajo con base en el conocimiento de sus experiencias previas; sin embargo, este nivel de reflexión es limitado, pues para dar solución a los problemas los profesores se apoyan en sus rutinas, en las sugerencias de sus colegas e incluso imitando a sus mejores profesores. Las reflexiones que se ofrecen desde este nivel de reflexión tienen un carácter casuístico y no teórico-conceptual; sin embargo, es una dimensión no menos importante, tanto así que a este nivel de reflexión le subyacen los planteamientos pedagógicos de Dewey, Claparède, Rafael Ramírez, Kerschensteiner y Neill, quienes resaltaron la necesidad de que los profesores reflexionaran desde la experiencia.

b) Reflexión técnica. Refiere al uso de conocimientos científicos teórico-pedagógicos provenientes de las ciencias de la educación, implica superar la reflexión práctica experiencial mediante la fundamentación de la solución de los problemas de la enseñanza en cuestiones y razonamientos científicos, utilizando procedimientos de carácter didáctico, así como metodologías aprobadas por el campo de la investigación en educación. Los aportes pedagógicos que sostienen la pertinencia de esta dimensión técnica son los del pensamiento de Claparède y Dewey, que fueron los pedagogos representantes de la corriente que reivindicó la racionalidad técnica para la pedagogía.

c) Reflexión social. Está compuesta por dos dimensiones: (1) la sociofuncional, la cual expresa el compromiso social y ético del profesor teniendo como fin la formación de sujetos socialmente funcionales y útiles; Dewey, Claparède y Kerschensteiner fueron los pedagogos partidarios de este nivel de reflexión. Y (2) la sociocrítica, que lleva inmersa la teleología de la educación, refiere a una reflexión donde los profesores muestran su compromiso social y ético, perciben la educación como un factor imprescindible en el diseño del humano, consideran el quehacer docente como una vocación para desarrollo personal y el cambio social. Los planteamientos pedagógicos de Rafael Ramírez estuvieron especialmente dirigidos a este tipo de reflexión, así como también los de Neill.

\section{MÉTODO}

Es una investigación de carácter cualitativo, narrativa y cuasiexperimental; se considera un estudio de casos múltiple (Yin, 1994; Stake, 1998), pues la muestra es de tipo intencional o por conveniencia y con base en las características de los sujetos participantes. 


\section{SUJETOS Y ESCENARIOS}

Los sujetos participantes de este estudio fueron 27 profesores que imparten clases en las licenciaturas de geodesia, geomática y astronomía de la Facultad de Ciencias de la Tierra y el Espacio, perteneciente al colegio de Ciencias Naturales y Exactas de la Universidad Autónoma de Sinaloa, México. 19 son hombres y 9 mujeres; 9 han concluido la licenciatura; 8, la maestría; 9, doctorado; y uno es pasante de doctorado. 15 profesores cuentan con entre uno y tres ańos de servicio; 8 , entre 4 y 15 ańos; y 4, con más de 16 ańos.

Los profesores tienen formaciones diversas. 16 son ingenieros topógrafos geodestas de la propia Universidad; 2 son ingenieros en sistemas; dos de informática; uno, ingeniero agrónomo; uno de matemáticas; uno de arquitectura; una profesora es astrónoma; $y$, últimamente, debido a cambios introducidos en el diseño curricular, se han incorporado dos psicólogas y una educadora para atender las asignaturas de ética y desarrollo profesional y lectura, redacción y comprensión de textos científicos.

De los 27 profesores se seleccionaron tres casos por considerarlos más representativos en la presencia de las tres dimensiones de la reflexión profesional: práctica, técnica y social; además, los diferencian los años de servicio docente en la institución, pues una de las profesoras cuenta con dos años, otro con 10 y otro más con 26 años de servicio; se les asignó la nomenclatura de Profesora A, Profesor B y Profesor C. Los datos de los profesores participantes se presentan en la tabla 1.

\begin{tabular}{cccc}
\hline \multicolumn{4}{c}{ TABLA 1. DATOS DE LOS PARTICIPANTES } \\
\hline Sujeto & Formación inicial & Nivel De estudios & ANTigüEDAd \\
\hline Profesora A & Psicología & Maestría & 2 años \\
\hline Profesor B & Ingeniería geodésica & Maestría & 10 años \\
\hline Profesor C & Ingeniería geodésica & Doctorado & 26 años \\
\hline
\end{tabular}

\section{INSTRUMENTOS PARA LA RECOLECCIÓN DE LA INFORMACIÓN}

Para la recolección de datos se utilizaron una diversidad de herramientas, en congruencia con la investigación narrativa planteada por Conelly \& Clandinin (1994); los instrumentos se enumeran a continuación:

1. Un programa de desarrollo profesional orientado a la educación del pensamiento profesional para generar la información empírica requerida.

2. La escritura de cartas docentes (Jacobo, Armenta, 2010 y 2014; Jacobo, Armenta, Ibarra, 2014). 
3. El uso de la wiki ${ }^{1}$ inscrita en el entorno virtual de aprendizaje alojado en la plataforma EDU20.

4. Registro diario de observación participante de las acciones llevadas a cabo en el taller de reflexión profesional.

5. Un sistema de categorías para el análisis de la información recabada.

En cuanto al mencionado sistema de categorías, se basa en el utilizado en otros estudios que indagaron sobre le efectividad de la wiki como herramienta para la sistematización de buenas prácticas pedagógicas (Jacobo, Hernández, Armenta, Ibarra, Gastélum, Muñiz, Rivera, 2011; Jacobo, Armenta, Ibarra, 2015; Jacobo, Ibarra, Armenta, 2016; Jacobo, Ibarra, Armenta y Gastélum, 2017). Sin embargo, en esta investigación se ha realizado una adaptación que desenfoca el uso de la wiki a favor de la prevalencia de la reflexión profesional como objeto de análisis. Tal sistema de categorías se organizó de forma abductiva con base en las tres dimensiones de la reflexión profesional, las cuales se describen enseguida.

En la dimensión práctica se espera encontrar fragmentos textuales que se refieran a las actividades y acciones que llevan a cabo en el aula, identificar en los que reciben acompańamiento y reorganizan sus ideas para reconstruir un guion de trabajo pedagógico mejor elaborado y detallado.

En la reflexión técnica se busca analizar las composiciones textuales donde los profesores pongan de relieve conocimientos teórico-pedagógicos que fundamenten las acciones descritas en su guion; el acompańamiento recibido por sus colegas y la reflexión colectiva, además del ejercicio de metacognición desarrollado por los profesores para hacer explícitas las teorías de las que parte su intervención e identificarlas en el guion de trabajo pedagógico, permitirán reorganizar su texto de manera radical, pues las antiguas ideas han sido abandonadas por nuevas a partir del estudio de las teorías del aprendizaje.

Por último, en la dimensión social se espera encontrar fragmentos textuales en los cuales se hagan presentes el compromiso ético y moral de los docentes; además, con el proceso reflexivo realizado y el acompañamiento recibido de los colegas, se espera que ajusten su guión de trabajo psicológico.

A continuación, se exponen las categorías y su descripción; seguido de ello, la adaptación de las categorías y la reorganización realizada en la tabla 2.

${ }^{1}$ La wiki es una herramienta digital que forma parte de la web 2.0 y permite la escritura y producción de textos en línea de manera tanto individual como colectiva, favorece la interacción entre grupos y crea un historial de registros en distintas versiones secuenciales. 


\begin{tabular}{|c|c|c|}
\hline \multicolumn{3}{|c|}{ TABLA 2. SISTEMA DE CATEGORÍAS PARA EL ANÁLISIS DE LA INFORMACIÓN } \\
\hline 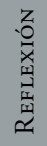 & Categorías de anÁlisis & DESCRIPCIÓN \\
\hline \multirow{2}{*}{ 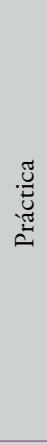 } & Acompañamiento entre pares & $\begin{array}{l}\text { Clasifica los segmentos narrativos en los que los colegas acompañan } \\
\text { en la identificación de los momentos por los que atraviesa el trabajo } \\
\text { pedagógico realizado, según lo descrito en la Experiencia Pedagógica } \\
\text { Exitosa. }\end{array}$ \\
\hline & $\begin{array}{l}\text { Guion de trabajo pedagógico. } \\
\text { Ajustes a la reflexión práctica }\end{array}$ & $\begin{array}{l}\text { Registra las modificaciones hechas por el docente a su experiencia } \\
\text { pedagógica exitosa a partir de las observaciones de sus pares y las } \\
\text { conversaciones colectivas traduciéndolo en la descripción de la se- } \\
\text { cuencia de acciones del trabajo pedagógico. Se espera que los textos } \\
\text { experimenten una nueva organización por cambio flexible (NOCF); } \\
\text { transformaciones morfosintácticas (TMFS) y transformaciones } \\
\text { semánticas por inclusión y supresión (TSI y/o TSS). }\end{array}$ \\
\hline \multirow[b]{2}{*}{ 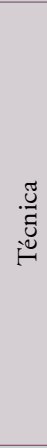 } & Acompańamiento entre pares & $\begin{array}{l}\text { Acompañamiento para la reflexión técnica (A_RT): se refiere a las } \\
\text { intervenciones escritas por los colegas que se acompañan entre sí } \\
\text { para traducir los conceptos básicos de las ciencias de la educación a } \\
\text { estrategias de trabajo pedagógico. }\end{array}$ \\
\hline & $\begin{array}{l}\text { Guion de trabajo pedagógico. } \\
\text { Ajustes a la reflexión técnica }\end{array}$ & $\begin{array}{l}\text { Registra las modificaciones hechas por el docente al guion de trabajo } \\
\text { pedagógico resultado de la reflexión sobre la práctica en la que docu- } \\
\text { menta la experiencia a partir de una teoría o teorías que den sustento } \\
\text { a su interacción didáctica en el aula. Se espera que, producto de la } \\
\text { reflexión individual y acompañamiento entre pares, el texto revele } \\
\text { Nueva Organización por Cambio radical (NOCR) y Transforma- } \\
\text { ciones Morfosintácticas, así como Transformaciones Semánticas por } \\
\text { Inclusión y Transformaciones Semánticas por Supresión. }\end{array}$ \\
\hline \multirow[b]{2}{*}{$\begin{array}{l}\bar{\pi} \\
\tilde{0} \\
\dot{\delta}\end{array}$} & Acompañamiento entre pares & $\begin{array}{l}\text { Acompańamiento para la reflexión social (AR_S): clasifica los } \\
\text { segmentos donde los colegas se acompañan entre sí para adecuar el } \\
\text { trabajo pedagógico a los fines sociales de la educación y el diseño de } \\
\text { lo humano. }\end{array}$ \\
\hline & $\begin{array}{l}\text { Guion de trabajo pedagógico. } \\
\text { Ajustes a la reflexión social }\end{array}$ & $\begin{array}{l}\text { Registra los ajustes hechos por el docente a su guion de trabajo } \\
\text { pedagógico a partir de la reflexión sobre el diseńo de lo humano en } \\
\text { la intervención pedagógica. Se espera que, producto de la reflexión } \\
\text { individual y acompañamiento entre pares, el texto revele Nueva } \\
\text { Organización por Cambio radical (NOCR) y Transformaciones Mor- } \\
\text { fosintácticas, así como Transformaciones Semánticas por Inclusión y } \\
\text { Transformaciones Semánticas por Supresión. }\end{array}$ \\
\hline
\end{tabular}

La clasificación de la información recabada se realizó mediante el uso del software Atlas-ti, versión 7.5.4, utilizado principalmente para el análisis de datos cualitativos. Este proceso permitió reducir la información a unidades de significado interpretables (unidades hermenéuticas). 


\section{PROCEDIMIENTO}

El taller de reflexión profesional tuvo un carácter de acompañamiento pedagógico y se organizó en seis módulos, los cuales se enuncian a continuación:

1. Aprender a navegar en el entorno virtual de aprendizaje.

2. La comprensión sistémica y compleja del conocimiento profesional docente.

3. La reflexión práctica. Una discusión conceptual.

4. La reflexión práctica. Documentar la experiencia.

5. La reflexión técnica en la intervención pedagógica.

6. La reflexión social en la intervención pedagógica.

El primer módulo tuvo lugar en la primera sesión presencial, se inició exponiendo a los profesores participantes la propuesta metodológico-didáctica del taller y sus propósitos. Los participantes aprendieron a navegar en la plataforma educativa EDU20, realizando de manera autónoma acciones como dar inicio y término a una sesión de trabajo virtual, ingresar a su cuenta y modificar sus datos personales, operar cada uno de los comandos del menú de trabajo, revisar las lecciones de cada módulo y su respectiva agenda de trabajo, lo mismo que enviar las tareas acordadas en la comunidad, utilizar con sentido las herramientas de chat y wiki y también debatir por ese medio.

En el segundo módulo los profesores revisaron los conceptos y principios de la teoría de la complejidad y de la teoría general de sistemas. Luego de aprender a navegar en el Entorno Virtual de Aprendizaje (EVA) y reflexionar sobre los macroconceptos de la sistémica y la complejidad para comprender de un modo diferente el conocimiento profesional docente, se procedió a hacer una revisión conceptual de la reflexión profesional en su dimensión práctica con base en los materiales de Donald Schön (1998) sobre epistemología de la práctica y el concepto de guion de Shank y Abelson (1987). Una vez realizada la revisión conceptual se desarrolló la actividad de composición de cartas docentes. El ejercicio consistió en recuperar una experiencia pedagógica exitosa (EPE) con la cual los profesores se sintieran identificados emocionalmente, ya sea por la actividad o estrategia didáctica realizada y/o por los logros obtenidos. La carta debía ser dirigida a un colega de confianza; ese primer ejercicio se registró en la wiki del EVA.

Durante el tercer módulo, que consistió en reconstruir la experiencia, los profesores desarrollaron y consolidaron las habilidades adquiridas en el módulo anterior, hicieron análisis de sus cartas para reconocer las estrategias intuitivas de composición utilizadas, los profesores analizaron sus cartas de manera individual y colectiva, de tal modo que cada uno de ellos revisó al menos una carta de sus colegas y una vez corregidas por sus autores, se realizaron observaciones colectivamente, dando como resultado la reconstrucción de lo que se da en llamar prosa de saber profesional; tal texto se presentó con ideas más claras y precisas.

Para efecto de realizar las observaciones a sus colegas, se les proporcionó una tabla en la que se indicaban los niveles de procesamiento y los descriptores de contenidos con base en los cuales hacer las sugerencias, preguntas o dudas que se desa- 
taran en su lectura; los niveles de procesamiento y los descriptores de contenido se presentan en la tabla 3.

\begin{tabular}{|c|c|}
\hline \multicolumn{2}{|c|}{ TABLA 3. NIVELES DE PROCESAMIENTO Y DESCRIPTORES DE CONTENIDO } \\
\hline $\begin{array}{c}\text { Nivel de } \\
\text { Procesamiento }\end{array}$ & DESCRIPCIÓN \\
\hline $1 .^{\circ}$ & Contexto de la acción pedagógica y caracterización de los sujetos o aprendices \\
\hline $2 .^{\circ}$ & Propósitos o contenidos de aprendizaje \\
\hline $3 .^{\circ}$ & Hipótesis de trabajo pedagógico \\
\hline $4 .^{\circ}$ & Ajuste según la reflexión práctica \\
\hline $5 .^{\circ}$ & Ajuste según la reflexión técnica \\
\hline $6 .^{\circ}$ & Ajuste según la reflexión social \\
\hline 7. & Referencias bibliográficas \\
\hline
\end{tabular}

En síntesis, en este primer acercamiento a su experiencia pedagógica exitosa, los participantes hicieron explícito su guion de trabajo pedagógico con base en la experiencia. Ello permitió que los profesores retomaran su carta docente para reelaborar un nuevo documento que trascendiera sus experiencias previas en el aula y los procesos de enseñanza cotidianos, con predominio de la abstracción reflexionante. Para la reelaboración de ese nuevo documento se les solicitó explícitamente que abandonaran el formato de carta para que fuera tomando la estructura de un documento profesional dirigido a otros profesionistas afines al área de la educación. En esta parte los profesores analizan y cuestionan su trabajo con base en sus experiencias previas en el aula y los procesos de enseńanza de la realidad pedagógica; de igual manera, se realizaron exposiciones para intercambiar y recibir opiniones y sugerencias, característica de la reflexión práctica. Vale decir que las reflexiones de los profesores en este nivel tuvieron un carácter casuístico y no teórico-conceptual. Al final reconocieron -a pregunta expresa- que sus prácticas docentes no se basaban en alguna teoría pedagógica o de la educación.

En el quinto módulo entró en juego el ejercicio de la reflexión técnica; con ese fin, se les proporcionaron a los participantes materiales de estudio referentes a las principales teorías del aprendizaje con base en las cuales podían hacer explícita su intervención en el aula, luego que confesaron no saber a qué teoría respondía su acción pedagógica; también realizaron búsquedas estratégicas de información sobre las mencionadas teorías en las bases de datos disponibles en la propia Universidad. Socializaron entre ellos la teoría del aprendizaje significativo de Ausubel; el aprendizaje cooperativo de Johnson \& Johnson; el aprendizaje por conflicto y descubrimiento con base en las ideas de Piaget; el aprendizaje sociocultural con base en las ideas de Vygotsky, entre otros. Identificaron en su quehacer docente algunas de las ideas planteadas en dichas teorías de forma no ortodoxa. Es decir, no necesariamente su intervención pedagógica se alineaba a una sola teoría, sino que tomaban de cada una la que se adecuaba mejor a las explicaciones desde y sobre la acción. Fue un 
momento donde los profesores trascendieron el carácter práctico de su experiencia redactada, es decir, incluyeron la base científica en su práctica educativa.

Una vez identificadas las teorías predominantes en la intervención pedagógica y documentadas en la wiki, se procedió a la revisión entre pares para hacer sugerencias y preguntas. Ello dio como resultado un documento en el que las expresiones emocionales fueron desvaneciéndose a favor de una prosa de saber profesional con transformaciones morfosintácticas y semánticas por inclusión y/o supresión que dieron lugar a nuevas organizaciones por cambio conceptual radical y/o cambio conceptual flexible.

En cuanto a la dimensión social de la reflexión profesional, se desarrolló durante el sexto y último modulo; los contenidos de los materiales estuvieron enfocados en desarrollar conversaciones en torno al compromiso ético y moral de los docentes. Es por eso por lo que se reflexionó sobre la visión y misión construida por el colectivo de profesores de la propia escuela, al mismo tiempo que se estudió el emblemático libro Carta a una profesora, escrito por los niños de Barbiana, para que intentaran transferir lo dicho ahí con la realidad de sus propios alumnos. Las conversaciones desatadas por esas lecturas permitieron a los docentes hacer explícito su compromiso con el desarrollo de lo humano, que se expresó en las últimas versiones de la wiki, tanto en las observaciones entre pares como en las correcciones hechas por los profesores.

\section{RESULTADOS Y ANÁLISIS DE CASOS}

Se presentan tres casos de los profesores seleccionados, el criterio de selección se basó en considerar que los profesores cubrían las tres dimensiones de la reflexión profesional: práctica, técnica y social. En cada caso se realiza el análisis de los resultados de acuerdo a cada dimensión de la reflexión profesional y el acompańamiento entre pares dispensado a los profesores por parte de sus colegas.

En la búsqueda de comprender y caracterizar los distintos conocimientos hechos explícitos por los profesores en las tres dimensiones, también durante el análisis estos conocimientos se asociaron de acuerdo a sus características a los distintos saberes que conforman el conocimiento profesional docente.

\section{Caso 1. Profesora A}

Profesora A es una profesora universitaria que tiene como grado académico inicial la licenciatura en psicología, además de ser estudiante de doctorado en educación; imparte las asignaturas de Ética y Desarrollo Profesional y Lectura, Redacción y Comprensión de Textos Científicos. Su área de especialidad e investigación es la Educación. Vale decir que durante sus primeros dos años como docente ha recibido el acompańamiento de una profesora experimentada. En los primeros dos momentos del procedimiento redactó en formato de carta dirigida a su amigo en la que relata una experiencia que consideró la más enriquecedora, motivo por el cual 
fue su elección. El proceso de sistematización de la experiencia la llevó a producir un total de catorce versiones en la wiki.

\section{Reflexión práctica}

El ejercicio de reflexión correspondiente a la dimensión práctica tiene su inicio a partir del registro de un guion inicial de clase donde de manera breve expone una serie de acciones; se registra tal y como lo escribió Profesora A:

Comparto el guion que realizo durante una sesión de clases en el curso de Ética y Desarrollo Profesional.

Saludo.

Activación del conocimiento previo sobre el tema.

Explico la dinámica de sesión.

Desarrollo de la sesión.

Valoración del nuevo conocimiento.

Retroalimentación grupal.

Tarea (a consideración).

Es observable que no existe una explicación detallada de las acciones del guion. Sólo consigna los grandes momentos de su intervención en el aula. En el análisis de los nuevos registros textuales se identifica el acompañamiento para la reflexión práctica (A_RP) brindado por un colega docente en el taller:

Estimada compañera, me parece muy apropiada la estrategia que sigues [...]. Aseguras que el estudiante tenga una verdadera experiencia cognoscitiva [...]. Sin embargo, ayudaría que lo aplicaras a un tema más especifico que pueda exponerse en una sesión. Mencionando también los instrumentos didácticos de los que te auxilias.

Se observa la sugerencia realizada al trabajo de Profesora A, se le indica que las acciones que describe en su guion pedagógico deberían estar orientadas a un tema en específico y no sólo a las acciones que realiza como profesor en una clase en general. En este mismo acompańamiento se le sugiere que mencione los instrumentos didácticos, es decir, los materiales y recursos de los que se apoya en su práctica docente.

Como resultado de la reflexión individual, colectiva y el acompañamiento recibido, se registran los ajustes hechos de Profesora A a su guion de trabajo pedagógico, en el que se puede percibir una descripción detallada de cada secuencia de acciones:

SAludo. En este momento, además de brindar un buen día o una buena tarde a los estudiantes les pregunto como amanecieron o cómo va su día. La intención en esa pregunta es animarlos y que sientan que son importantes en mi clase, así como la actitud que ellos tengan en ese momento también es importante. 
Activación del conocimiento previo sobre el tema. Considero importante conocer el conocimiento previo que tienen los estudiantes sobre el tema a revisar, será su punto de vista, quizá con información de sentido común. En este sentido, el alumno podrá incorporar conocimiento científico asociado con el existente, de esta forma podría ser más fácil incorporar los nuevos conceptos.

EXPLICO LA DINÁMICA DE SESIÓN. Describir cómo abordaremos el tema, las actividades y que es lo que pretendemos con ello. De esta forma el estudiante tiene una idea de lo que realizaremos y lo que se espera al final de la sesión.

Desarrollo de la sesión. Consiste en la implementación de las actividades planteadas anteriormente, que se muestren con buena actitud e interés los estudiantes. VAloración del nuevo conocimiento. Podría hacerse de distintas formas, como realizar un tipo sondeo y que la participación sea voluntaria o específica. De esta forma habría un acercamiento al conocimiento que se adquirió durante la sesión. Retroalimentación Grupal. Para los jóvenes es muy atractivo el trabajo en grupo, aunque aquí no sería trabajo en equipos, es un análisis grupal de lo que se ha revisado en la sesión, de esta forma los estudiantes aportan sus ideas, con un lenguaje más cotidiano, así como los ejemplos para que vuelva más sencillo el aprendizaje. TAREa (a CONSIDERACión). Lo manejaría a consideración, por la cuestión de los temas. Quizás no sea necesario una tarea, en caso de dejarla consistirá en un ejercicio donde el alumno haga una reflexión sobre los temas revisados durante la sesión y cómo lo aplicaría en su vida profesional.

Por lo que se puede percibir, reelabora su documento recurriendo a sus experiencias previas en el aula para así registrar una nueva versión, describiendo y detallando las distintas acciones o actividades que realiza en la clase, es decir, reflexionando desde lo práctico para explicar el procedimiento realizado.

También es notorio cómo Profesora A, en el inicio de su guion, muestra preocupación por el estado de ánimo de los estudiantes, considerando importante conocerlo como factor que incide en el aprendizaje, esto refiere a lo que Shulman (1987) expone como el conocimiento de los alumnos y de sus características, un tipo de conocimiento que, de acuerdo a este mismo autor, deriva de la sabiduría que la misma práctica les otorga a los docentes. Otro fragmento muestra cómo Profesora A realiza la actividad de explicar la dinámica de la sesión, describiendo a los estudiantes las actividades a desarrollar, a este tipo de conocimiento Tardif (2004) lo denomina saber experiencial, que tiene su génesis y desarrollo en el trabajo cotidiano del profesor hasta tomar forma de rutinas y habilidades.

En este caso, se presenta en el pensamiento de Profesora A una Nueva Organización por Cambio Flexible (NOCF), pues sólo agrega más precisiones al guion de trabajo pedagógico con base en la reflexión práctica. Además, se presentan Transformaciones Semánticas por Inclusión (TSI) de nuevas ideas. 
En su reflexión inicial Profesora A busca fundamentar las acciones realizadas en su clase, en razones y conocimientos teórico-pedagógicos provenientes de las ciencias de la educación, identificadas con el aprendizaje significativo de Ausubel:

Desde la teoría del aprendizaje significativo desarrollo mi práctica pedagógica al consultar entre los estudiantes la noción que tienen sobre el tema o ciertos conceptos claves que se revisarán durante la sesión...

En el registro para el acompañamiento a la reflexión técnica (A_RT) una colega hace el siguiente comentario a Profesora A:

Estimada compañera, me parecen interesantes y acertados tus comentarios sobre la aplicación de los conceptos de aprendizaje al impartir el curso. Me llama la atención sobre cómo se genera el aprendizaje cooperativo. Si es el caso, como mencionas, que también se aplica el aprendizaje significativo, «sería interesante cómo se da en algún o algunos conceptos importantes de la ética y desarrollo profesional. Esto es en qué caso específico de la materia se pueden generar los aprendizajes de recepción, inclusión y descubrimiento».

Saludos.

La nueva composición de texto muestra al inicio una explicación de la teoría del aprendizaje de Ausubel, exponiendo algunos conceptos clave de dicha teoría; este ejercicio permite que el profesor vaya construyendo un conocimiento propio de la enseñanzañ. Para Elbaz (1981) es un conocimiento de la instrucción, referido a las técnicas de enseñanza desde un sustento teórico. Por su parte Porlán, Rivero y del Pozo (1997) lo señalan como un conocimiento académico, definiéndolo como saberes pedagógicos y didácticos que tienen por objeto la enseñanza de los contenidos escolares. Es por eso por lo que se identifican argumentaciones que se organizan en ideas más precisas y claras sobre la teoría que ha elegido para fundamentar su práctica docente; una nueva organización por cambio radical (NOCR) es visible:

De acuerdo a la teoría del aprendizaje significativo desarrollado por Ausubel et al. (1983), este se genera mediante dos condiciones por recepción y por descubrimiento. [...] El aprendizaje por recepción se da mediante tres tipos: 1) Aprendizaje de representaciones [...], 2) Aprendizaje de conceptos [...] y 3) Aprendizaje de preposiciones [...]. El aprendizaje por descubrimiento; en este tipo de aprendizaje significativo el alumno descubre por él mismo, generando proposiciones soluciones a problemas planteados.

Lo que también se expresa en un incipiente guion de trabajo pedagógico con ajustes para la reflexión técnica:

«En el momento de mi guión, activación del conocimiento previo sobre el tema aplico la teoría del aprendizaje significativo». En primer momento generando el aprendizaje por recepción para que los alumnos relacionen el nuevo conocimiento con el existente en su estructura cognoscitiva. 
«Durante el momento de valoración del nuevo conocimiento, se pregunta de forma azarosa o voluntaria a los estudiantes» quién desea participar con una idea central y/o punto de vista sobre el tema revisado. «Se desea activar en ese momento la reflexión de los estudiantes» mediante un análisis de la participación del compañero estudiante y lo revisado durante la clase.

En este caso la teoría de aprendizaje de Ausubel no fue la única fuente a la que acudió Profesora A para fundamentar su práctica, sino que también percibió que podía incluir la teoría del aprendizaje cooperativo, por lo que teniendo en cuenta las características de sus acciones precisó por lo pronto los componentes invariantes del aprendizaje cooperativo en los siguientes fragmentos narrativos:

La teoría del aprendizaje cooperativo desde la perspectiva de los autores Johnson y Johnson (1999) fomenta el trabajo en equipo y resta importancia a la competitividad entre los estudiantes, así como la búsqueda en lograr gran productividad solo en algunos sujetos.

De acuerdo con los autores mencionados anteriormente, el aprendizaje cooperativo está comprendido en cinco componentes básicos que a continuación se mencionan:

- La interdependencia positiva; consiste en lograr que cada uno de los participantes en un grupo escolar sepa que el trabajo grupal fortalece al individual.

- La Responsabilidad individual y grupal; este componente pretende adquirir como grupo la responsabilidad para cumplir los objetivos previstos compartiendo entre cada uno de los integrantes una parte de la tarea, valorando y evaluando el esfuerzo y desempeño de cada uno de los miembros.

- La interacción social; esta promueve entre los alumnos la motivación para lograr el éxito, estimulándose unos a otros, de manera que los miembros del grupo adquieren un compromiso personal y grupal para cumplir de manera satisfactoria con la tarea propuesta como grupo de trabajo.

- La interacción personal; consiste en estrategias grupales que tienen por objeto mantener activos a cada uno de los integrantes. Todos deben saben cómo actuar cuando haya dudas y conflictos, saber cómo deben organizarse para cumplir una tarea y cómo promover la motivación en el grupo para continuar con ella.

- La evaluación grupal; valora si los objetivos previstos se lograron no solo como grupo cooperativo, sino también por cada uno de los que participan en él.

Se aprecia cómo en sus intentos por fundamentar y explicar desde la teoría de aprendizaje significativo de Ausubel y el aprendizaje cooperativo de Johnson \& Johnson, las distintas versiones van reconstruyéndose.

En el análisis a la reflexión sobre la práctica se puede apreciar un intento por fundamentar técnicamente su trabajo pedagógico, se hacen visibles Nuevas Organizaciones por Cambio Radical en las ideas escritas en la wiki, pues en las últimas versiones introduce conceptos que no aparecían en las iniciales; pese a ello y a los ajustes, el guion de trabajo pedagógico no varió significativamente del que planteó desde la reflexión práctica, lo cual puede deberse a la dificultad de traducir el saber disciplinar a estrategias de trabajo pedagógico. 
Es en la última versión de la wiki en la que se registra el ejercicio de reflexión de tipo social, una actividad en la que el profesor debe mostrar su interés por el diseño de lo humano, el futuro de sus estudiantes y el tipo de sociedad que busca formar desde las aulas. Cabe señalar que para esta dimensión de la reflexión profesional no recibió retroalimentación de sus colegas.

A continuación, se muestra la composición textual registrada en esta versión:

Como docentes debemos de tener actitudes congruentes con el comportamiento que reflejamos durante nuestra práctica docente y fuera de ella [...] «Para los estudiantes es importante, la persona que está frente a ellos y el desempeńo que demuestre en su profesión, es un ejemplo que puede relacionar con su posible éxito profesional». De acuerdo a las aptitudes que deben de contar los estudiantes al momento de ser egresados de la FACITE, «es esencial generar y fortalecer durante las sesiones las participaciones individuales en el sentido de desarrollar en el estudiante un pensamiento reflexivo para su crecimiento profesional y personal». Sin duda, la aplicación de trabajo en equipo desarrolla en los estudiantes habilidades de trabajo colaborativo, manejo de emociones, retroalimentación, interacción interdisciplinar.

Los ajustes al guion tampoco se vieron reflejados en la secuencia de acciones en esta dimensión. Aunque se observa cómo a través de este ejercicio se toma en cuenta el éxito profesional de los estudiantes donde las actitudes congruentes de la práctica docente son un factor importante en tal logro, además se expresa la pertinencia de desarrollar el pensamiento reflexivo en los estudiantes con el fin de fortalecer su crecimiento profesional y personal. Estos planteamientos reflejan lo que Shulman (1987) plantea como el conocimiento de los objetivos, las finalidades y los valores educativos.

Pese a los cambios percibidos en los diferentes niveles de reflexión, Profesora $\mathrm{A}$ aún tiene dificultades para traducir a los grandes momentos de la enseñanza los conceptos de las ciencias de la educación. Esto podría explicarse por la falta de experiencia que caracteriza su condición de profesora principiante.

\section{Caso 2. Profesor B}

Los registros que evidencian la reflexión práctica de Profesor B se aprecian desde la primera versión, en la que presenta una descripción del proceso y actividades que desarrolla en la clase, recurriendo a sus experiencias previas y algunas de sus rutinas con sus estudiantes, brinda una explicación desde el trabajo práctico que realiza con sus alumnos:

FASE INICIAL

1. Punto de encuentro en el almacén de instrumental geodésico a la hora programada.

2. Saludo inicial y pase de lista por brigada.

3. Traslado al lugar elegido para realizar la actividad. 


\section{Desarrollo}

4. Reunión para realizar un resumen general de la actividad a desarrollar y explicar las condiciones bajo las cuales será realizada y evaluada la misma.

5. Demostración in situ de la actividad de mi parte de manera detallada de cada una de las fases del proceso de nivelado y centrado del teodolito electrónico.

6. Sesión de aclaración de dudas respecto al proceso demostrado.

7. Repetición de la o las fases, que lo requieran según la percepción de la actividad del punto anterior.

8. Desarrollo de la actividad de manera individual por parte de cada integrante de la brigada.

9. Realización de rondas de visita a cada brigada para verificar que la actividad se encuentre desarrollando en orden y para resolver dudas o problemas en el desarrollo de la misma.

\section{ConClusión}

10. Reunión final con el objetivo de consultar sobre los detalles que resultaron más complejos en el proceso y resumir mediante participación colectiva lo más destacado de la actividad.

11. Indicaciones sobre la forma de realizar el reporte correspondiente y tiempo de entrega.

12. Despedida.

Dado que gran parte de las actividades declaradas en el perfil de egreso de los estudiantes se refiere a actividades de campo, esto sin duda hace de este tipo de cursos una experiencia especial. Comparto esta experiencia que me ha resultado gratificante y a mi juicio exitosa, con el objetivo de recibir retroalimentación de tu parte.

Profesor B, a diferencia de Profesora A, desde su primer registro derivado de la reflexión práctica, enumera una serie de acciones con su respectiva descripción de las actividades que realiza en clase. Además, divide su guion pedagógico en momentos, el cual tiene un inicio, desarrollo y conclusión. De acuerdo a la descripción que realiza, demuestra tener conocimiento del contenido (Marcelo, 2005; Shulman; 1987), es decir, propio de la asignatura que enseña y del uso de los instrumentos que utiliza. Otras acciones descritas como el saludo, aclarar dudas, dar distintas indicaciones en distintos momentos, se han constituido para Profesor B como una serie de guiones y rutinas (Porlán, Rivero y Martín, 1997) que le ayudan a resolver distintos acontecimientos que ocurren en su trabajo pedagógico.

Enseguida del registro del guion detallado se identifica un fragmento de acompañamiento para la reflexión práctica (A_RP):

Estimado Amigo, te saludo con gusto y me permito anotar algunos comentarios al escrito de arriba:

* El proceso de la clase está sumamente detallado, lo que lo hace útil como referencia para distintas clases similares, experimentales u observacionales.

* Algunos puntos pueden hacerse más concisos, como, por ejemplo: «5. Demostración in situ de la actividad de mi parte de manera detallada de cada una de las fases del proceso de nivelado y centrado del teodolito electrónico», puede resumirse a algo como " 5 . Demostración in situ de las fases del proceso de 
nivelado y centrado del teodolito electrónico». Pero es una sugerencia mínima, con fines de facilidad una lectura rápida.

El acompañamiento recibido le sugiere al profesor realizar transformaciones semánticas y morfosintácticas al texto que contribuyan a su reorganización para darle más coherencia o lógica a la redacción. Después se vuelve a presentar otro texto que indica nuevamente acompańamiento para la reflexión práctica (A_RP):

Estimado profesor, respecto a su guion de trabajo comento que está muy completo. Por otro lado, como la materia de instrumental geodésico, es una materia teórica-practica, como muchas otras, «en este tipo de materias se aplica en gran sentido el aprendizaje cooperativo, donde los alumnos establecen relaciones para llegar a un objetivo común, el cual es el realizar sus prácticas de una manera satisfactoria y el aprender el cómo se realizan los procesos para la misma». Sin más me despido, felicitándolo y deseándole que esté bien.

El nuevo acompañamiento le sugiere al profesor que de acuerdo a las características de su guion puede ser pertinente fundamentarlo en los principios del aprendizaje cooperativo, un señalamiento que indica la necesidad de buscar un sustento teórico pedagógico. Como resultado de las observaciones se presenta nuevamente una NOCF con algunas nuevas modificaciones que señalan nuevos momentos que detallan más el guion:

FASE INICIAL

1. Punto de encuentro en el almacén de instrumental geodésico a la hora programada.

2. Saludo inicial y pase de lista por brigada.

3. Traslado al lugar elegido para realizar la actividad.

FASE DE DESARROLLO

Instrucción

4. Reunión para realizar un resumen general de la actividad a desarrollar y explicar las condiciones bajo las cuales será realizada y evaluada la misma.

Modelado

5. Demostración de la actividad de mi parte de manera detallada de cada una de las fases del proceso de nivelado y centrado del teodolito electrónico.

Reforzamiento

6. Sesión de aclaración de dudas respecto al proceso demostrado.

7. Repetición de la o las fases, que lo requieran según la percepción de la actividad del punto anterior.

\section{Ejecución}

8. Desarrollo de la actividad de manera individual por parte de cada integrante de la brigada. 


\section{Supervisión}

9. Realización de rondas de visita a cada brigada para verificar que la actividad se encuentre desarrollando en orden y para resolver dudas o problemas en el desarrollo de la misma.

\section{Retroalimentación}

10. Reunión final con el objetivo de consultar sobre los detalles que resultaron más complejos en el proceso y resumir mediante participación colectiva lo más destacado de la actividad.

\section{FASE DE CONCLUSIÓN}

11. Indicaciones sobre la forma de realizar el reporte correspondiente y tiempo de entrega.

12. Despedida

\section{Reflexión técnica}

En el ejercicio correspondiente a la reflexión técnica Profesor B optó por fundamentar su práctica en la teoría del aprendizaje sociocultural de Vygotsky, del cual algunas ideas ya se percibían desde la reflexión práctica. De este modo presenta las distintas acciones que describe en su guion pedagógico, dándose a la tarea de buscar los conceptos clave para precisar y buscar nuevas ideas en la fundamentación técnica de su quehacer docente:

Apoyándome en la teoría del paradigma educativo sociocultural definido por Vigotsky, realizo la reflexión técnica del guion de la clase Proceso de centrado y nivelado del teodolito electrónico.

En la fase de desarrollo declarada en el guion en cuestión, se pueden ubicar los componentes de este paradigma, en primera instancia, la identificación de la ZDPot (Zona de Desarrollo Potencial) del alumno, misma que se encuentra señalada en el propósito declarado en el Programa de Estudios de la Unidad de Aprendizaje correspondiente.

La ZDR (Zona de Desarrollo Real) del alumno, según Vigotsky, está conformada por la condición socio-cultural y de conocimientos previos que presenta el alumno en el momento de tener la experiencia educativa, elementos necesarios para facilitar la apropiación del conocimiento.

El andamiaje diseńado para poder generar la transición de la ZDR a la ZDPot, se encuentra definida en los pasos 5 al 9 de la fase de desarrollo descrita en el guion. Dada la asimetría natural en la que se da el proceso educativo, lo que exige tomar en la fase inicial la dirección, lo que se hace evidente en el paso 5 en el que realizo la demostración práctica de las habilidades que deberán adquirir los alumnos en forma de conocimiento.

Naturalmente en la primera interacción demostrativa los resultados de la comprensión de los procesos presentados no son asimilados con el mismo nivel por todos los alumnos, y considerando lo que sostiene Baquero respecto que el andamiaje debe ser ajustable, los pasos 6 y 7 atienden esta propiedad, con el objetivo de generar la 
ZDP (Zona de Desarrollo Próximo) en el alumno, y al mismo tiempo atendiendo lo que Onrubia sostiene: «la vinculación continua entre lo dado y lo nuevo es un recurso característico de la construcción de las ZDP».

Dentro de los criterios establecidos por el mismo Onrubia, para la intervención para que la asistencia otorgada a los alumnos logre un aprendizaje significativo en ellos, establece que debe procurarse el uso autónomo y autorregulado de los contenidos por parte de los alumnos, lo que se observa en el paso 8 del guion.

Mientras que en el paso 9, se atiende otro criterio de Onrubia, que sostiene que es fundamental la interacción entre los alumnos como otro recurso valioso para la creación de la ZDP, lo que comparte Vigotsky, el cual, considera que sean también los pares más capaces quienes puedan participar para promover zonas de construcción. Respecto al proceso de la evaluación en este paradigma, Vigotsky propone la evaluación dinámica, la cual se basa en la evaluación del producto, pero haciendo énfasis en los procesos en desarrollo, lo que de manera implícita se describe en el paso 9.

Profesor B redacta de manera detallada la fundamentación de cada una de las acciones de su guion basándose en los conceptos clave de la teoría del aprendizaje sociocultural, este ejercicio le permitió ir construyendo y haciendo explícito un saber profesional que, de acuerdo a los planteamientos de Tardif (2004), es un saber de carácter pedagógico que se produce en la investigación realizada en el área de las ciencias de la educación; sin embargo, de acuerdo a la explicación de Profesor B en el conocimiento no solamente del contenido, sino también de los alumnos y sobre la teoría del aprendizaje, es un saber más complejo, un conocimiento psicopedagógico, que se relaciona con los saberes sobre los principios generales de la enseñanza, el aprendizaje y los alumnos (Marcelo, 2005).

Lo escrito por Profesor B revela una Nueva Organización por Cambio Radical en sus ideas tanto como en la traducción de las mismas en el guion de trabajo pedagógico con base en la reflexión técnica y Transformaciones semánticas por inclusión (TSI).

\section{Reflexión social}

En la parte final del documento registrado textualmente por Profesor B se localiza una serie de fragmentos identificados como una NOCR, donde muestra el impacto de la dimensión social de la reflexión en su guion pedagógico mediante tres puntos donde explica la forma en la que su guion aporta al cumplimiento del diseño de lo humano en los estudiantes:

Respecto al impacto en la dimensión social del guion al que hago referencia en este ejercicio, tomo como base lo declarado institucionalmente en el perfil de egreso del estudiante del programa educativo de ingeniería geodésica [...]

La forma en que en este guion aporto a que se cumpla la dimensión social en el estudiante es la siguiente:

Punto 1: Mostrará actitudes de ética, honestidad e interés por la constante actualización en su campo profesional. 
En este caso, de manera general se trabaja en la formación de principios de conducta fundamentales, tales como el respeto, la honestidad, la responsabilidad, otros.

$[\ldots]$

Punto 2: Tendrá habilidades de manejo de personal y de trabajo en equipo.

La aportación a este punto se da propiciando condiciones en las que sea necesario formar grupos de trabajo y realizar actividades de manera coordinada, donde el grado de responsabilidad recae de manera equitativa en los integrantes del grupo y asignando la dirección de la actividad a un integrante en particular, haciendo un cambio de rol frecuentemente.

Punto 3: Mostrará respeto al medio ambiente y/o nivel de concientización ante el impacto ambiental.

La naturaleza de la profesión en un índice significativamente alto, se desarrolla en contacto con el medio ambiente, las clases de carácter práctico, permiten con mayor significancia desarrollar una formación conductual con responsabilidad del espacio con que se interactúa (no generar basura o en su defecto colectarla y depositarla donde corresponde, no dañar la vegetación sin necesidad o el menor de los dańos posibles, etc.).

Por último, Profesor B recibe acompañamiento para la reflexión social de un colega docente que expresa su impresión por el trabajo tan detallado y desarrollado:

Profesor

Agradezco un desarrollo tan detallado en su reflexión social sobre la contribución que usted logra mediante sus clases a favorecer el perfil del egresado. Comparto que efectivamente los valores y virtudes que deseamos inculcar en nuestros estudiantes deben ser observados claramente en el profesor para poder ser transmitidos.

También me ha interesado que efectúe un cambio de rol de los estudiantes en el trabajo en equipo ya que eso favorece a la formación de las habilidades llamadas «blandas», pero que son sumamente importantes en el desarrollo profesional de los muchachos, en este caso, liderazgo, capacidad de comunicación, colaboración e integración.

Finalmente, adecuadamente el respeto al entorno es de esperarse en egresados de nuestro campo, aunque no implica que sea innato. De forma que es conveniente reforzarlo.

Una felicitación por la forma en que explícitamente realiza esta reflexión.

Se puede percibir en Profesor B, a lo largo del desarrollo de la reflexión profesional plasmada en la WIKI, la capacidad intelectual para aplicar los conceptos de las ciencias de la educación en los momentos del guion de trabajo pedagógico con base en la reflexión técnica y la reflexión social, haciéndose explícitas las ideas que le subyacen. Lo siguiente sería ver qué tanto de su conocimiento declarativo es posible verlo traducido en la planificación de la clase e implementación en el aula, propiamente hablando, lo cual no es objeto de estudio de este trabajo, por cierto. 


\section{Caso 3. Profesor C}

Profesor $\mathrm{C}$ es un profesor universitario que tiene como grado académico la ingeniería en geodesia como formación inicial, cuenta con 26 años de trabajo docente, posee la maestría y doctorado en ciencias geodésicas, además de ser investigador en la propia FACITE. La sistematización de su práctica pedagógica conlleva el objetivo de hacer explícita su intervención pedagógica en la enseñanza de la asignatura de geodesia física.

\section{Reflexión práctica}

En un primer momento Profesor $\mathrm{C}$ registra una serie de acciones que remiten a las actividades que realiza en una clase más centradas en el contenido de su asignatura:

1. Recordar el concepto de funciones armónicas visto en clase anterior y preguntar al respecto.

2. Mencionar la naturaleza de las soluciones de las funciones armónicas.

3. Describir los elementos de la fórmula.

4. Analizar el comportamiento de algunos componentes de la fórmula.

5. Evaluar la fórmula numéricamente.

6. Mostrar gráficos.

7. Explicar la tarea correspondiente.

8. Preguntas.

Enumera los distintos momentos característicos de un guion, ese es el conocimiento explícito acerca de su clase, que, desde la perspectiva de Porlán, Rivero y Martín (1997), son saberes basados en la experiencia que refieren a un conjunto de ideas de carácter consciente y que los profesores utilizan en su práctica docente; son saberes que tienden a orientar la conducta de los profesores en los momentos de programación y evaluación de la clase.

Siguiendo la evolución del guión de Profesor C se presentan dos momentos de acompañamiento a la reflexión práctica, de manera secuencial por dos docentes distintos:

«Sería importante que al iniciar su clase les diera el saludo a sus alumnos, para darles la confianza e iniciar su clase». Por otra parte, me parece interesante la manera que estructura su clase, porque así, el alumno se adentra en el tema, y con ello, tener un mejor aprovechamiento del tema.

Se observa cómo se le sugiere un gesto de amabilidad hacia sus estudiantes para establecer un ambiente de confianza en el aula, incluyendo el saludo al iniciar su clase. 
Más adelante, en otra versión de su wiki, se le sugiere que describa algunas experiencias sobre cómo aplicar lo que enseña en la clase, en la que resalta la pertinencia de describir con detalle el guion redactado inicialmente.

Hola estimado compañero, es de gran utilidad para mí haber leído el guion que seguiste en tu clase, uno de los aspectos importantes en la comprensión de las clases es el interés que los alumnos tienen para aprender el contenido, es de decir, las ganas que ellos le pongan a lo que quieren aprender. «Por lo tanto, algo importante es el poner o indicar algunas experiencias o problemas que se pueden aparte del indicado, es decir, indicar como puedo aplicar lo aprendido».

Sin más me despido agradeciendo su atención y deseándole lo mejor.

Como resultado del proceso de reflexión y el acompañamiento recibido, Profesor $\mathrm{C}$ registra cambios significativos en sus ideas, pues aparece en el nuevo texto la figura del estudiante, aunque aún implícita, en los momentos 1, 4, 6, 10, 11 y 12 , es decir, 6 de los 12 momentos consignados en el trabajo de su clase, los cuales se refieren a saludar, preguntar, dar información, cuestiones que no había considerado en su guion inicial, lo cual revela una Nueva Organización por Cambio Radical en sus ideas. La secuencia del guion se enumera enseguida:

1. Saludar a la clase y dar los buenos días.

2. Recordar el concepto de funciones armónicas visto en clase anterior y preguntar al respecto.

3. Mencionar la naturaleza de las soluciones de las funciones armónicas, empleando el pizarrón.

4. Describir los elementos de la fórmula, proyectando el material en formato digital. Preguntar si hay dudas al respecto.

5. Analizar el comportamiento de algunos componentes de la fórmula, proyectando el material en formato digital.

6. Preguntar si hay dudas con respecto a lo expuesto.

7. Evaluar la fórmula numéricamente. Para esto se emplea el programa matemático MathCad por el proyector.

8. Mostrar gráficos de la evaluación de la fórmula mediante el programa MathCad por el proyector.

9. Se entrega el material de la tarea correspondiente en impreso.

10. Se da información de ayuda para resolver la tarea, y referencias para que el estudiante pueda abundar más sobre el tema.

11. Sesión de preguntas sobre el tema y tareas.

12. Informar sobre el tema de la siguiente clase y despedirse. 
En esta dimensión de la reflexión, Profesor C generó un fragmento textual en el que reconoce la teoría de Ausubel como generadora del aprendizaje en sus estudiantes. Tal afirmación se puede leer en el siguiente fragmento:

Generación del Aprendizaje

En esta clase el principal aprendizaje es de tipo significativo, ya que el estudiante afianza el conocimiento previamente adquirido. Esto es, comprende de una mejor manera el concepto de las funciones armónicas con aplicación a la teoría del potencial terrestre. Asimismo, el conocimiento por representación se genera al poder representar y apreciar los gráficos de las funciones involucradas. Por otro lado, se espera que el alumno obtenga el aprendizaje por inclusión al asociar las funciones armónicas como elementos de la geometría analítica o del análisis funcional. Finalmente, el aprendizaje por descubrimiento se produce al experimentar y aplicar las fórmulas numéricamente, lo que a su vez coloca al estudiante en posición de dar el paso a las proposiciones en la aplicación del conocimiento adquirido.

En este caso, no se presentó retroalimentación, ni traducción de los saberes pedagógicos a la intervención didáctica que se viera reflejada en el guion, aunque se puede considerar que la conversación colectiva generó que investigara sobre el aprendizaje significativo y lo asociara a su intervención pedagógica.

\section{Reflexión social}

En el final de su documento profesional el profesor registra algunas ideas que permiten afirmar que el compromiso ético y social del profesor implica generar en los estudiantes conocimientos para que sean bien aplicados por el futuro profesional, tal como se puede leer en el siguiente párrafo:

La enseńanza del presente tema contribuye al perfil de egreso del ingeniero geodesta en los aspectos:

Realización de levantamientos de apoyo vertical. Al aplicar la fórmula en modelos geopotenciales para obtener alturas geoidales.

Poseerá conocimientos de matemáticas y física necesarios para una mejor comprensión, desarrollo y aplicación de la ingeniería geodésica.

Contará con los conocimientos necesarios que le permitan laborar en los niveles de planeación, organización, dirección y ejecución en las tareas de cartografía, geodesia, topografía y fotogrametría.

Se promueve la actitud ética y honestidad profesional al recomendar aplicar adecuadamente y en forma correcta las ecuaciones tratadas.

Conocerá metodologías y conocimientos de la geodesia superior para ejecutar obras ingenieriles con alta precisión cuidando el impacto ambiental y de riesgos.

Para esta dimensión de la reflexión profesional, aunque se refleja el compromiso ético por formar profesionistas honestos, es probable que la ausencia de retroalimentación entre pares no hiciera visibles los cambios en las ideas de Profesor C. 


\section{DISCUSIÓN Y CONCLUSIONES}

El objetivo de la investigación fue documentar el ejercicio de reflexión profesional de profesores universitarios del área de ciencias poniendo a prueba un programa de desarrollo profesional en comunidad de práctica con base en el acompañamiento entre pares, con la planta docente de la Facultad de Ciencias de la Tierra y el Espacio de la Universidad Autónoma de Sinaloa, para hacer explícitas las dimensiones o saberes que componen el conocimiento profesional del profesor, saberes de tipo práctico, pedagógico y ético-social. El ejercicio hizo posible visibilizar el guion de trabajo pedagógico de los docentes universitarios.

En los casos de Profesora A y Profesor C, sólo se perciben avances en el guion de trabajo pedagógico con base en la reflexión práctica; en el caso de Profesor $\mathrm{B}$, en las tres dimensiones de la reflexión profesional.

En cuanto a los cambios percibidos en la reestructuración de las ideas de los profesores por Nueva Organización por Cambio Flexible y Nueva organización por cambio radical, fue posible percibir que en los tres se hizo visible en las versiones de la wiki en que recibieron acompañamiento de sus pares; en cambio, las reestructuraciones por NOCF y NOCR no se percibieron donde dicho acompañamiento estuvo ausente.

Se percibió en mayor medida cierta dificultad para ofrecer acompañamiento en la dimensión social de la reflexión profesional, probablemente por la dificultad que entraña traducir el compromiso ético y moral en algún momento específico del guion de trabajo pedagógico.

En relación con los años de servicio de los profesores estudiados, se perciben dificultades para traducir a estrategias de intervención didáctica en Profesora A -considerada principiante- y Profesor C, considerado como experimentado. En cambio, en Profesor B, se percibe cierta facilidad para traducir el saber de las ciencias de la educación al guion de trabajo pedagógico con base en la reflexión técnica y social, aunque, cabe señalar, es quien más recibió acompañamiento de sus colegas, mientras que para Profesora A y Profesor C, fue menos frecuente.

El análisis de los casos permitió comprender que el conocimiento profesional de los profesores es construido mediante la reflexión y la socialización de experiencias educativas exitosas con otros colegas, esto demuestra la capacidad de los profesores como profesionistas reflexivos y como sujetos de saber. El conocimiento profesional implica la puesta en marcha de distintos saberes tanto prácticos como técnicos entendidos estos últimos como de tipo científico. El trabajo en colectivos docentes demuestra un aspecto importante en la formación profesional y continua de los profesores universitarios, por lo que es necesario que los profesores participen en programas de desarrollo profesional tendientes a aplicar las teorías de la educación que contribuyan a generar conocimiento profesional que pueda ser replicado por las nuevas generaciones de profesionales. 


\section{REFERENCIAS BIBLIOGRÁFICAS}

Alumnos de la Escuela de Barbiana (1975). Carta a una profesora. México: Ediciones de Cultura Popular.

Angulo, J.F. (1999). «De la investigación sobre la enseñanza al conocimiento docente», en PÉREz, A., Gómez, Barquín Ruiz, J. y Angulo Rasco, J.F. (eds.). Desarrollo profesional del docente. Política, investigación y práctica (pp. 261-319). Madrid: Akal.

Clarà, M. y Mauri, T. (2010). «El conocimiento práctico. Cuatro conceptualizaciones constructivistas de las relaciones entre conocimiento teórico y práctica educativa». Infancia y Aprendizaje, 33(2), 131-141.

Connelly, M. y Clandinin, J. (1995). "Relatos de experiencia e investigación narrativa», en Larrosa, J. (ed). Déjame que te cuente. Ensayos sobre narrativa y educación (pp. 1-25). Barcelona: Laertes.

Dávila, G., Leal, F., Comelin, A., Parra, M. y Varela, P. (2013). «Conocimiento práctico de los profesores: sus características y contradicciones en el contexto universitario actual». Revista de la Educación Superior, 42(166), 35-53.

Dewey, J. (1989). Cómo pensamos. La relación entre pensamiento reflexivo y proceso educativo. México: Paidós.

Elbaz, F. (1981). «The Teacher’s «Practical Knowledge»: Report of a Case Study». Curriculum Inquiry, 11(1), 43-71. Doi: 10.1080/03626784.1981.11075237.

Gamboa, A. (2017). «Enseńanza universitaria y conocimiento profesional docente». Ventana Cientifica, 8(13), 29-42.

GutiérRez, Y. (2012). «La investigación sobre el conocimiento del profesor y sus perspectivas para el estudio de concepciones didácticas y disciplinares en la enseñanza de la lengua materna», en Soler Castillo, S. (comp.). Lenguaje y educación: Aproximación desde las prácticas pedagógicas (pp. 79-106). Bogotá: CADE.

Hurtado E.A., Serna, A.M. y Madueño, S.M. (2015). «Práctica docente del profesor universitario: su contexto de Aprendizaje. Profesorado». Revista de Curriculum y Formación de Profesorado, 19(2), 215-224.

Imbernón, F. (2004). La formación y el desarrollo profesional del profesorado. Hacia una nueva cultura profesional (6. ed.). España: Graó.

Jacobo García, H.M., Armenta Beltrán, M., Ibarra Aguirre, E. (2015). «Educación del pensamiento profesional en ambientes virtuales. La wiki como herramienta para sistematizar buenas prácticas pedagógicas», disponible en http://www.comie.org.mx/congreso/memoriaelectronica/v13/doc/2856.pdf.

Jacobo García, H.M., Hernández Vaca, S., Armenta Beltrán, M., Ibarra Aguirre, E., Hernández Gastelum, C.J., Rivera Calvo, E. y Muñiz, S. (2011). «La escuela normal de la experiencia. Sistematizacion de buenas prácticas pedagógicas de profesores de educación básica», en Congreso Iberoamericano de profesores que hacen investigación desde la escuela, realizado en Argentina en julio de 2011.

Jасово, H.M. (2006). «Teorías del desarrollo en el saber de los profesores. El papel de los programas de desarrollo profesional», еn Јасово, H.M. (ed.). Educación y formación de profesores. Complejidad cognitiva y entorno global (pp. 161-212). Barcelona-México: Pomares. 
Jacobo, H.M. (2009). El profesionalismo integrado. Un nuevo modo de ser educador. México: Ed. Plaza y Valdés.

Jacobo, H.M., Ibarra, E. y Armenta, M. (2016). «Una aproximación teórica-metodológica a la sistematización de buenas prácticas de aprendizaje servicio». Educación y Diversidad, 10(2), $101-114$.

Jacobo, H.M., Ibarra, E., Armenta, M. y Gastelum, C.J. (2017). «La wiki como herramienta para sistematizar buenas prácticas pedagógicas». Revista de investigación en tecnologías de la información, 5(9), 7-15.

Johansen, B. (1992). Introducción a la teoría general de sistemas. México: Editorial Limusa.

MarCelo, C. (2005). «La investigación sobre el conocimiento de los profesores y el proceso de aprender a enseñar: una revisión personal», en Perafán, A. y Adúriz, A. (comp.). Pensamiento y conocimiento de los profesores. Debate y perspectivas internacionales (pp. 45-61). Bogotá: Nomos.

Marrero, J. (1993). «Las teorías implícitas del profesorado: vínculo entre la cultura y la práctica de la enseñanza», en Rodrigo, M.J., Rodríguez, A. y Marrero, J. (eds.). Las teorías implícitas. Una aproximación al conocimiento cotidiano (pp. 243-270). Madrid: Visor.

Marrero, J. (2009). El pensamiento reencontrado. Espańa: Editorial Octaedro.

Morin, E. (2002). La mente bien ordenada. Barcelona: Seix-Barral.

Pérez, A.I. y Gimeno, J. (1988). «Pensamiento y acción en el profesor: de los estudios sobre la planificación al pensamiento práctico». Infancia y Aprendizaje, 42, 37-63.

Pérez-Gómez, A. (2010). «La naturaleza del conocimiento práctico y sus implicaciones en la formación de docentes». Infancia y Aprendizaje: Journal for the Study of Education and Development, 33(2), 171-177. DOI: 10.1174/021037010791114652.

Pérez, A.I., Soto, E. y Serván, M.J. (2015). «Lesson Studies: re-pensar y re-crear el conocimiento práctico en cooperación». Revista Interuniversitaria de Formación del Profesorado, 84(29.3) 81-101.

Porlán, R., Rivero, A. y Martín, R. (1997). «Investigación didáctica. Conocimiento profesional y epistemóloga de los profesores I: teoría, métodos e instrumentos». Enseñanza de las ciencias, 15(2), 155-17.

Rodríguez, J. y Alamilla, P. (2018). «La complejidad del conocimiento profesional docente y la formación del conocimiento práctico del profesorado». Actualidades Investigativas en Educación, 16(2), 1-24. DOI: 10.15517/aie.v18i2.33129.

SснӧN, D.A. (1992) La formación de profesionales reflexivos. España: Paidós.

SснӧN, D.A. (1998). El profesional reflexivo. Cómo piensan los profesionales cuando actúan. México: Paidós.

Shank y Abelson (1987). Guiones, planes, metas y entendimiento. Barcelona: Paidós.

Shavelson, R. y Stern, P. (1981). «Investigación sobre el pensamiento pedagógico del professor, sus juicios, decisions y conductas», en Gimeno Sacristán, J. y Pérez Goméz, A.I. (comps.). La enseñanza: su teoría y su práctica (pp. 372-419). Madrid: Akal.

Shulman, L.S. (1987). «Knowledge and Teaching: Foundations of the New Reform». Harvard Educational Review, 57(1), 1-22. DOI: 10.17763/haer.57.1.j463w79r56455411.

Stake, R.E. (1998). Investigación con estudios de caso. Madrid: Morata.

TARDIF, M. (2004). Los saberes del docente y su desarrollo profesional. Madrid: Narcea.

YIN, R.K. (1994). Investigación sobre estudio de casos. Diseños y Métodos. London: SAGE. 
